\title{
Programa Bolsa Família: Uma Análise Espacial nos Municípios do Paraná em 2004, 2008 e 2012
}

\author{
Marcos Aurélio Brambilla ${ }^{1}$ \\ Marcio Marconato ${ }^{2}$ \\ Márcia Regina Gabardo da Camara ${ }^{3}$ \\ Sergio Carlos Carvalho ${ }^{4}$ \\ Sidnei Pereira do Nascimento ${ }^{5}$
}

\begin{abstract}
Resumo: O objetivo do trabalho é verificar a relação entre a taxa de pobreza e o benefício médio do Programa Bolsa Família nos anos de 2004, 2008 e 2012 nos municípios paranaenses, a fim de confirmar se o programa está focalizado nos municípios com maiores taxas de pobreza. Para isso, o trabalho utiliza como metodologia a Análise Exploratória dos Dados Espaciais e analisa o coeficiente I de Moran bivariado, o gráfico de dispersão de Moran bivariado e o mapa de cluster das variáveis dos municípios. Os dados foram extraídos nos sites do Ministério de Desenvolvimento Social e Combate à Fome (MDS) e do Instituto Brasileiro de Geografia e Estatística (IBGE). Os resultados do estudo mostram que os municípios paranaenses com elevada taxa de pobreza exibiam maiores valor do benefício médio do Programa Bolsa Família, por outro lado, as unidades espaciais com baixo índice de pobreza apresentam baixo valor do benefício do Programa Bolsa Família. O estudo permite concluir que ao longo do período houve aumento da autocorrelação espacial indicando que ocorreu uma expansão do programa no Paraná, alcançando as regiões mais pobres do estado.
\end{abstract}

Palavras-chave: Programa Bolsa Família. Taxa de pobreza. Benefício médio.

JEL: J18

1- Mestre em Economia Regional pela Universidade Estadual de Londrina (UEL). E-mail: marcos-brabilla@ hotmail.com

2- Doutorando em Teoria Econômica pela Universidade Estadual de Maringá. E-mail: marconatoce@bol. com.br

3 - Doutora em Economia pela Universidade de São Paulo (USP). Professora titular da Universidade Estadual de Londrina. E-mail: mgabardo@sercomtel.com.br

4 - Doutor em Economia Aplicada pela Escola Superior de Agricultura 'Luiz de Queiroz' - Universidade de São Paulo (Esalq-USP). Professor titular da Universidade Estadual de Londrina. E-mail: sc.carvalho@terra. com.br

5- Doutor em Economia Aplicada pela Escola Superior de Agricultura 'Luiz de Queiroz' - Universidade de São Paulo (Esalq-USP). Professor titular da Universidade Estadual de Londrina. E-mail: sidnei@uel.br 


\section{Bolsa Família Program: A Spatial Analysis in Paraná Municipalities in 2004,2008 and 2012}

ABSTRACT: The aim of the research is to verify the relation between the poverty rate and the average annual benefit of a cash transfer program from Federal Government Bolsa Família Program for cities in Paraná state, in the years of 2004 2008 and 2012, to confirm that the program focalizes the cities where poverty is concentrated. In this regard, this study applies the Exploratory Spatial Data Analysis methodology to identify Moran's bivariate coefficient, scatterplots, significance maps and, finally, cluster variables maps of the cities with data provided by the Ministry of Social Development and Fight Against Hunger (MDS) and Brazilian Institute of Geography and Statistic (IBGE). The study allows to conclude that the increment of average benefit (BFP) in cities with high poverty rate and low benefit in cities with low poverty rate as well as the expansion of the program in Parana now reaching the poorest regions in the state.

Key-words: Bolsa Família Program. Poverty Rate. Average benefit.

JEL: J18

\section{INTRODUÇÃO}

Durante a década de 90 foram implantados no Brasil os programas de transferência de renda com o objetivo de reduzir as altas taxas de pobreza. No ano de 2003, durante a gestão do governo o Lula, houve a unificação de diversos programas assistenciais ${ }^{1}$ criados no governo Fernando Henrique Cardoso e assim surgiu o Programa Bolsa Família (PBF).

O Programa Bolsa Família visa contribuir para a inclusão social de milhões de famílias brasileiras em situação de miséria, com alívio imediato de sua situação de pobreza e da fome. Além disso, também busca estimular o acompanhamento do público-alvo nos serviços de saúde e ajudar a superar indicadores ainda dramáticos, que marcavam as trajetórias educacionais das crianças mais pobres, altos índices de evasão, repetência e defasagem idade-série. Pretende-se, assim, contribuir para a interrupção do ciclo de reprodução da pobreza (CAMPELLO, 2013).

O programa busca atender famílias de baixa renda em situação de vulnera1.Programa Bolsa Escola, Bolsa Alimentação, Cartão alimentação e Auxílio Gás. bilidade, segundo o Ministério do Desenvolvimento Social (MDS, 2015) o PBF atendeu mais de 14 milhões de famílias em todo o Brasil no ano de 2014. O elevado contingente de pessoas vivendo na pobreza é um dos principais problemas que o Brasil enfrenta atualmente no âmbito social e econômico. Logo, é necessária a intervenção do governo com políticas de transferência direta de renda para garantir o essencial dessa população mais vulnerável (PIRES; LONGO, 2008).

O estado do Paraná é uma das 27 unidades da federação, situado na região Sul, e possui a sexta maior população do país e o quinto maior Produto Interno Bruto (PIB) a preços constantes (IBGE, 2015). Entretanto, o estado paranaense apresentou em torno de 5,5\% dos seus domicílios em situação de pobreza no ano de 2013 (IPEA, 2015). O Programa Bolsa Família beneficiou aproximadamente 406 mil famílias paranaenses no ano de 2014 e repassou em torno de R \$ 697 milhões (MDS, 2015)

No entanto, para que um programa social seja capaz de reduzir a pobreza e a desigualdade ele precisa, antes de tudo, atingir a população que vive em condições de pobreza. Quanto maior a proporção dos recursos do programa alocados a esta população, maior será o seu grau de focalização e, consequentemente, maior o seu impacto sobre a pobreza e a desigualdade (BARROS et al., 2008).

Tos últimos anos, o país vem apresentando sinais de redução nos índices de pobreza e os programas de transferência direta de renda tem um papel importante para esse feito, principalmente o atual programa do governo, $o$ Programa Bolsa Família (JANNUZZI; BICHIR; RITZI, 2012). A partir desse contexto surgem algumas indagações: o programa tem surtido efeito, de modo que as cidades paranaenses mais pobres tenham um valor proporcional maior do benefício?

Lima, Caldarelli e Camara (2014) identificam nos anos 2000, clusters de subdesenvolvimento, que concentram a população mais pobre e as regiões menos desenvolvidas nas mesorregiões paranaenses Centro Sul e Centro Oriental. Neste sentido justifica-se a realização do presente artigo, que busca verificar se as regiões pobres e mais subdesenvolvidas do estado estão sendo atendidas pelo programa PBF. O tema é de grande importância para o Paraná e há poucos artigos que abordam o tema utilizando a abordagem de Análise Exploratória de Dados Espacial. O objetivo do presente trabalho é verificar a relação entre a taxa de pobreza e o benefício médio do Programa Bolsa Família (PBF) nos anos de 2004, 2008 e 2012 nos municípios paranaenses, a fim de verificar se o programa esta focalizado nos municípios com maiores taxa de pobreza.

O artigo está estruturado em seis partes. A primeira parte de natureza introdutória apresenta o contexto, o objetivo e a justificativa da pesquisa. A segunda discute a evolução das políticas sociais no Brasil, a terceira parte argumenta sobre os programas de transferência de renda, que aborda o Programa Bolsa 
Família, trazendo seus benefícios no começo do programa e atualmente, na quarta parte é apresentada a metodologia, na quinta é discutido os resultados da pesquisa e por último as principais conclusões.

\section{A EVOLUÇÃO DAS POLÍTICAS SOCIAIS NO BRASIL}

A situação de pobreza não se resume em apenas uma única definição, mas pode-se dizer que a pobreza se refere a uma condição em que determinada pessoa não consegue manter um padrão de vida satisfatório para arcar com suas necessidades básicas considerando um dado período (BARROS; HENRIQUES; MENDONÇA, 2001).

Na década passada, ocorreram grandes mudanças no que tange às políticas sociais do país, resultando em uma diversificação das estratégias para o combate à pobreza. $\mathrm{O}$ país atualmente conta com diversos programas sociais descentralizados, que caracterizam a política social brasileira atual (BARROS; CARVALHO, 2003).

Na maioria dos países dos países ocidentais, a noção de justiça social encontra-se definida nas constituições, com isso cabe ao Estado formular e implementar as políticas sociais. O desenvolvimento pleno das políticas sociais do Estado ocorre a partir do pós-guerra entre as décadas de 50 e 70. Na década de 70, os Estados nacionais, inclusive o brasileiro, vivenciaram crises fiscais e de endividamento interno e externo, após a crise do petróleo e do sistema financeiro internacional, que comprometem o financiamento de suas políticas sociais. Nos anos 1980 e 1990, os Estados nacionais para equacionarem suas crises fiscais e financeiras buscam alternativas de gestão para financiar as políticas públicas. Logo, para que se tenha uma boa administração pública é necessário que o processo político feito pelos participantes das áreas de decisão política seja de qualidade (FERNANDES, 2006)

Para Silva, Yazbek e Giovanni (2004), o Governo Fernando Henrique Cardoso teve como principal prioridade o ajuste e a estabilidade econômica, colocando as questões sociais do Brasil em um segundo plano. Apenas a partir de 20010 governo cria a "rede de proteção social", com programas nacionais destinados às famílias pobres, na categoria Renda Mínima, sendo o Bolsa-Escola a nível nacional acompanhado de outros programas nos níveis municipais e estaduais, que haviam começado em 1995, a exemplo do Programa de Garantia de Renda Familiar Mínima do município de Campinas/SP, programa adotado também pelo município de Ribeirão Preto/SP. Ainda em 1995, foram implementados o Programa Bolsa-Escola adotado em Brasília/DF e o Programa "Nossa Família" no município de Santos/SP.

O estudo realizado por Barros, Henriques e Mendonça (2001) mostra que o
Brasil, no início do século XXI não era um país pobre, mas sim um país com um grande número de pobres, e de muita injustiça e desigualdade. Um dos desafios da política social, seria a implementação de instrumentos de política econômica que ao mesmo tempo resultassem em maior eficiência econômica e justiça social.

\section{PROGRAMAS DE TRANSFERENCIA DE RENDA}

De acordo com Pires e Longo (2008), os programas de transferência de renda são programas sociais com uma intervenção do governo na economia, buscando resultados imediatos para combater a pobreza e a concentração de renda. Para isso é necessário que ocorra uma maior participação do setor privado nos programas sociais e crescente eficiência na alocação e na distribuição de recursos para garantir aos indivíduos o atendimento de suas necessidades básicas alimentares, permitindo a integração dos indivíduos na vida econômica moderna. Mas nem sempre os programas sociais adotados pelo governo alcançam os resultados esperado.

Os programas de transferência de renda recebem muitas críticas no que se refere ao "efeito preguiça" dos beneficiários, que por estarem recebendo uma remuneração, podem induzir à desmotivação dos membros e assim acarretam a diminuição da oferta de trabalho. Mas o desincentivo do trabalho é muito pequeno ou não existe, segundo os resultados de Oliveira e Soares (2012).

O trabalho realizado por Marinho, Linhares e Campelo (2011) mostra uma relação não significativa entre transferências de renda e pobreza, reforçando o argumento de autores críticos dos programas de transferência de renda, que falam da ineficiência, pelo fato de grande parte dos recursos não ser destinado às pessoas que estão realmente em situação de pobreza ou porque ocasiona uma dependência do benefício, o chamado fenômeno da armadilha da pobreza.

O estudo de Medeiros, Britto e Soares (2007) foca dois programas de transferência de renda, o Benefício de Prestação Continuada (BPC) e o Programa Bolsa Família (PBF) e conclui que os programas estão cumprindo a função a que se destinam e, aparentemente, não tem efeitos negativos sobre os incentivos para o mercado de trabalho.

De acordo com Lavinas (2006), há um aumento expressivo no valor destinado as políticas de sociais de 2001 a 2004, resultando, portanto, em uma redução considerável do número de pobres em 28\% de 2001 a 2004.

O estudo realizado por Jannuzzi, Bichir e Ritzi (2012) mostra que o PBF apresenta impactos positivos na condição de vida no que tange a educação e saúde, além de ter contribuído para a redução da população em situação de pobreza e extrema pobreza em $35 \%$. 
Conforme Soares e Sátyro (2010), desde a criação do PBF em outubro de 2003, a estrutura praticamente é a mesma. As informações referentes ao PBF são do Cadastro Único, que contêm informações da população pobre do país, mesmo sendo imperfeitas e incompletas. E não só são usadas pelo PBF, mas também por outros programas sociais do governo.

Segundo Monteiro (2008), o Programa Bolsa Família sugere, para o combate à fome e a pobreza as seguintes dimensões essenciais: transferência direta de renda para as famílias, para redução imediata da pobreza; ter os direitos sociais básicos de Saúdes e Educação melhor exercidos e a unificação de programas complementares para que as famílias consigam superar a pobreza e a extrema pobreza. O programa beneficia as famílias conforme sua renda mensal por pessoa e o número de crianças e adolescentes até 17 anos.

O PBF não é um direito, o programa depende diretamente da renda das famílias beneficiárias, ou seja, só recebe o benefício às famílias que atenderem a renda estipulada pelo programa, conforme a lei $n^{0} 10.833$, de janeiro de 2004 que instituiu o PBF e diz: o Poder Executivo deverá compatibilizar a quantidade de beneficiários do Programa Bolsa Família com as dotações orçamentárias existentes (SILVA; TAVARES, 2010).

Segundo Lavinas $(2006,2007)$ o aumento do gasto público social no Brasil, mediante o incremento das transferências monetárias sujeitas à comprovação de renda, contribuiu para reduzir a pobreza, mas o modelo de combate à pobreza adotada não foi acompanhado pelo crescimento simultâneo nos gastos per capita em educação, saneamento básico, habitação, logo não soluciona a questão da desigualdade.

Costa e Cunha (2014) verificaram o impacto do Programa Bolsa Família na oferta de trabalho dos beneficiários no Brasil e no Paraná. Os resultados encontrados pelas autoras mostram que o PBF impactou de forma positiva na participação no mercado de trabalho das mulheres, tanto das que são chefes de família, quanto das cônjuges; por sua vez, no Paraná, apenas no caso das mulheres cônjuges foram encontrados valores significativos. Nesse sentido, as autoras concluíram que o PBF não representa um fator de desestímulo ao trabalho, tendo em vista que a probabilidade de participação no mercado de trabalho não é reduzida para os beneficiários em grande parte dos subgrupos analisados.
De acordo com Cotta e Paiva (2010), o PBF é praticado com pelo menos dois benefícios desde seu início e apresenta dois tipos de linha de pobreza, que depende da renda familiar per capita. A Tabela 1 resume os valores do Programa Bolsa Família e sua evolução entre os anos de 2004 e de 2012.

No ano de 2012 foi criado o benefício de superação da extrema pobreza (BSP), que tem como proposta acabar com a extrema pobreza no país, pela sua característica, mesmo as famílias que receberem os outros benefícios do programa e que continuarem na situação de pobreza extrema (até R $\$ 70,00$ per capita), tem direito a receber um valor suficiente para superar essa situação (MDS, 2015)

TABELA 1: ELEGIBILIDADE DO PROGRAMA BOLSA FAMÍLIA - EVOLUÇÃO DE 2004 A 2012

\begin{tabular}{|c|c|c|c|c|c|c|c|c|}
\hline \multicolumn{3}{|c|}{ Critérios } & $\begin{array}{l}2004 \\
2005\end{array}$ & 2006 & 2007 & 2008 & $\begin{array}{l}2009 \\
2010\end{array}$ & $\begin{array}{l}2011 \\
2012\end{array}$ \\
\hline \multirow{2}{*}{\multicolumn{2}{|c|}{$\begin{array}{l}\text { Elegibilidades } \\
\text { Renda Familiar } \\
\text { Mensal Per Capita } \\
\quad \text { Pobreza }\end{array}$}} & $\begin{array}{l}\text { Extrema } \\
\text { Pobreza }\end{array}$ & 50,00 & 60,00 & 60,00 & 60,00 & 70,00 & 70,00 \\
\hline & & 100,00 & 120,00 & 120,00 & 120,00 & 140,00 & 140,00 & 140,00 \\
\hline \multirow{5}{*}{ Benefícios } & Básico & \multirow{3}{*}{$\begin{array}{l}\text { Extrema } \\
\text { Pobreza }\end{array}$} & 50,00 & 50,00 & 58,00 & 62,00 & 68,00 & 70,00 \\
\hline & Variável & & $\begin{array}{l}15,00 \\
(0-14)\end{array}$ & $\begin{array}{l}15,00 \\
(0-14)\end{array}$ & $\begin{array}{l}18,00 \\
(0-14)\end{array}$ & $\begin{array}{l}20,00 \\
(0-15)\end{array}$ & $\begin{array}{l}22,00 \\
(0-15)\end{array}$ & $\begin{array}{l}32,00 \\
(0-15)\end{array}$ \\
\hline & BVJ & & - & - & - & $\begin{array}{c}30,00 \\
(16- \\
17)\end{array}$ & $\begin{array}{c}33,00 \\
(16-17)\end{array}$ & $\begin{array}{c}38,00 \\
(16-17)\end{array}$ \\
\hline & Variável & \multirow{2}{*}{ Pobreza } & $\begin{array}{l}15,00 \\
(0-14)\end{array}$ & $\begin{array}{l}15,00 \\
(0-14)\end{array}$ & $\begin{array}{l}18,00 \\
(0-14)\end{array}$ & $\begin{array}{l}20,00 \\
(0-15)\end{array}$ & $\begin{array}{l}22,00 \\
(0-15)\end{array}$ & $\begin{array}{l}32,00 \\
(0-15)\end{array}$ \\
\hline & BVJ & & - & - & - & $\begin{array}{c}30,00 \\
(16-17)\end{array}$ & $\begin{array}{c}33,00 \\
(16-17)\end{array}$ & $\begin{array}{c}38,00 \\
(16-17)\end{array}$ \\
\hline
\end{tabular}

4. METODOLOGIA E BASE DE DADOS

O presente item apresenta a estratégia empírica da pesquisa. A metodologia está dividida em seis partes: análise exploratória de dados espaciais, matrizes de dados espaciais, estatística I de Moran Bivariada, diagrama de dispersão de Moran Bivariada, indicadores locais de associação espacial (LISA - Local Indicators of Spatial Association) e fontes de dados e descrição das variáveis. 


\subsection{ANÁLISE EXPLORATÓRIA DE DADOS ESPACIAIS
(AEDE)}

A Análise Exploratória de Dados Espaciais é a coleç̃o de técnicas para descrever e visualizar distribuições espaciais, identificar localidades atípicas (outliers espaciais), descobrir padrões de associação espacial (clusters espaciais) e sugerir diferentes regimes espaciais e outras formas de instabilidade (ANSELIN, 1999).

Segundo Almeida (2012), o primeiro passo no estudo da Análise Exploratória dos Dados Espaciais (AEDE) é testar a hipótese de que os dados espaciais sejam distribuídos aleatoriamente. Intuitivamente, aleatoriedade espacial significa que os valores de um atributo numa região não dependem dos valores deste atributo nas regiões vizinhas.

É importante ressaltar que, segundo Almeida (2012), essa análise é mais apropriada na investigação de variáveis espacialmente densas ou intensivas, ou seja, que são divididas por algum indicador de intensidade (per capita, etc.)

Desta forma, a partir da Análise Exploratória de Dados Espaciais é possível extrair medidas de autocorrelação espacial global e local, investigando a influência dos efeitos espaciais por intermédio de métodos quantitativos (ANSELIN, 1988). Contudo, antes de desenvolver a AEDE é necessário, previamente, estabelecer um arranjo que permita estimar coeficientes que deem a ideia do grau de interação entre as unidades espaciais (NETO; MEDEIROS, 2011).

\subsection{MATRIZES DE PESOS ESPACIAIS}

A dependência espacial é uma das características dos dados espaciais, pode se manifestar em diversos campos de estudo, como meio ambiente, na criminologia e também na economia, em variáveis como, por exemplo, desemprego. Entretanto, um aspecto fundamental na determinação da autocorrelação espacial é a consideração do grau de vizinhança o qual se deseja realizar a análise de dependência espacial. Uma vez adotado o critério de vizinhança, pode ser construída a matriz de pesos espaciais (SABATER; TUR; AZORÍN, 2011).

As matrizes de pesos espaciais são baseadas na contiguidade, que, por sua vez, pode ser definida de acordo com a vizinhanca. $\mathrm{O}$ grau de conexão expresso na matriz de peso espacial pode ser classificado de acordo com um critério geográfico. O critério de conexão geográfica da matriz de pesos espaciais apoia-se na ideia de proximidade, que, por sua vez, pode ser definida de acordo com a contiguidade e/ou com a distância geográfica (ALMEIDA, 2012). Na literatura as matrizes de pesos espaciais mais utilizadas são a matriz Rainha e Torre exibida na Figura 1.
FIGURA 1: MATRIZES DE PESOS ESPACIAIS

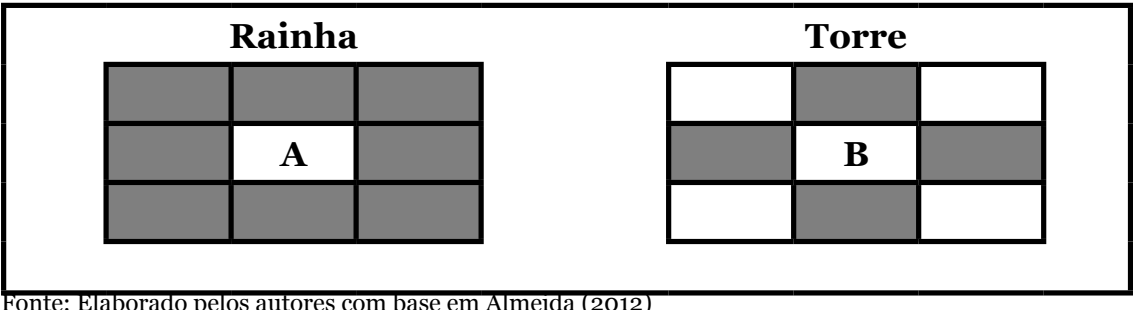

A convenção de contiguidade rainha (Figura 1) a região "A" tem como vizinhos oito regiões. Já, a matriz torre tem como vizinhos apenas as células destacadas em cor escura, sendo assim, apenas quatro regiões seriam consideras vizinhas de "B".

\subsection{A ESTATÍSTICA I DE MORAN BIVARIADA}

No estudo da Análise Exploratória dos Dados Espaciais (AEDE) é possível obter um coeficiente de autocorrelação espacial global num contexto bivariado. Segundo Almeida (2012), a ideia intuitiva é descobrir se o valor de um atributo observado numa dada região esta relacionado espacialmente com os valores de outra variável observada em regiões vizinhas. Assim é possível calcular a estatística $I$ de Moran para duas varáveis diferentes padronizadas, digamos, $Z_{1}$ e $Z_{2}$ neste caso obtemos a equação 1 :

$$
\mathrm{Iz}_{1} \mathrm{z}_{2}=\frac{\mathrm{Z}_{1}^{\prime} \mathrm{WZ}_{2}}{\mathrm{Z}_{\mathrm{\rho}}^{\prime} \mathrm{Z}_{1}}
$$

Em que $\mathrm{WZ}_{2}$ é a defasagem espacial da variável padronizada $\mathrm{Z}_{2}$, a matriz $W$ é normalizada na linha. De acordo com Anselin et al. (2003), essa estatística fornece o grau de associação linear (positiva ou negativa) entre o valor para uma variável em uma dada locação $i$ e a média de uma outra variável nas locações vizinhas $j$.

Segundo Almeida (2012), o I de Moran fornece três tipos de informações, o nível de significância prove a informação sobre os dados estarem distribuídos aleatoriamente ou não, o sinal positivo da estatística $I$ de Moran, desde que significativo, indica que os dados estão concentrados através das regiões, o sinal negativo, por sua vez, indica a dispersão dos dados e a magnitude da estatística fornece a força da autocorrelação espacial, quanto mais próximo de um mais forte é autocorrelação e quanto mais próximo de -1 mais disperso estão os dados. 


\subsection{DIAGRAMA DE DISPERSÃO DE MORAN BIVARIADO}

Segundo Almeida (2012), o diagrama de dispersão de Moran bivariado é uma alternativa para visualizar a autocorrelação espacial, o qual mostra a defasagem espacial da variável de interesse no eixo vertical e o valor de outra variável de interesse no eixo horizontal. De acordo com Diniz (2012), além da medida global de associação linear espacial o diagrama fornece outras informações, tais como quadrantes representando quatro tipos de associação linear espacial, a saber, Alto-Alto (AA), Baixo-Baixo (BB), Alto-Baixo (AB) e Baixo-Alto (BA) Um exemplo do diagrama de dispersão de Moran é apresentado na Figura 2 :

FIGURA 2: DIAGRAMA DE DISPERSÃO DE MORAN BIVARIADO

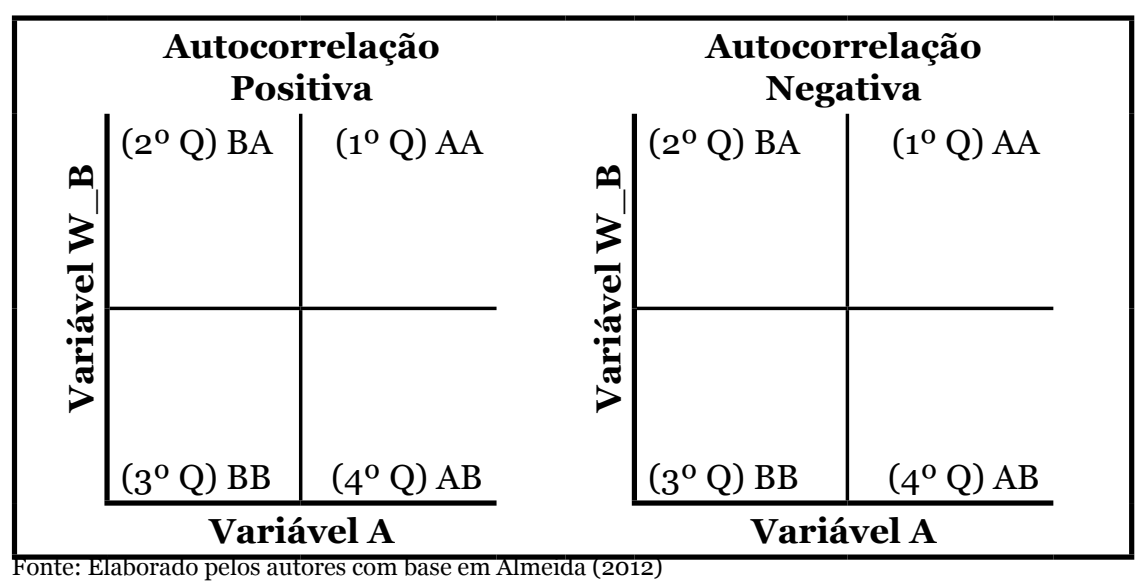

Um agrupamento Alto-Alto significa que as unidades espaciais pertencentes a esse agrupamento exibem valores altos das variáveis de interesse (A) rodeados por unidades espaciais que apresentam valores também altos de (W_B), representado pelo primeiro quadrante do diagrama. Um agrupamento Baixo-Baixo refere-se a um agrupamento cujas unidades espaciais mostram valores baixos da variável (A) circundados por unidades espaciais que ostentam valores também baixos de (W_B), representado pelo terceiro quadrante.

Um agrupamento Alto-Baixo diz respeito a um cluster no qual uma unidade espacial qualquer com um alto valor de uma das variáveis de interesse (A) e um baixo valor da outra variável defasada (W B). Isso é representado pelo quarto quadrante. Um agrupamento Baixo-Alto concerne a um cluster no qual uma unidade espacial qualquer com um baixo valor de uma variável de interesse (B) e um alto valor da outra variável defasada (W_B). Isso é representado no segundo quadrante.

\subsection{AUTOCORRELAÇÃO ESPACIAL LOCAL BIVARIADA}

Os indicadores LISA indicam o grau de autocorrelação espacial Local. Conforme Anselin (1995), para que isso ocorra é necessário que essa estatística satisfaça a dois critérios: a) esses indicadores devem possuir, para cada observação, uma indicação de clusters espaciais significantes de valores similares ao redor de cada observação, b) o somatório dos indicadores LISA, em todas as regiões, deve ser proporcional ao indicador de autocorrelação espacial global. Segundo Almeida (2012), o indicador de autocorrelação espacial bivariado local (LISA) pode ser obtido por intermédio da equação 2:

$$
\mathrm{I}_{\mathrm{i}} \mathrm{Z}_{1} \mathrm{z}_{2}=\mathrm{Z}_{1 \mathrm{i}} \mathrm{WZ} \mathrm{Z}_{2 \mathrm{i}}
$$

Em que $\left(\mathrm{WZ}_{\mathrm{p}}\right)$ é a defasagem espacial da variável padronizada $\left(\mathrm{Z}_{\mathrm{2}}\right)$ Assim somente os vizinhos da observação $i$, definidos conforme uma matriz de pesos espaciais são incluídos no cálculo. O indicador local de associação espacial fornece o grau de autocorrelação espacial estatisticamente significativo, em cada unidade regional (HADDAD; PIMENTEL, 2004).

Combinando as informações do diagrama de Moran bivariado com o mapa LISA de significância obtemos o mapa de cluster com os regimes bivariados, que permite uma visualização geográfica mais adequada do grau de concentração das variáveis estudadas.

De acordo com Anselin (1995), a estatística LISA, é usada para testar a hipótese nula, ou seja, a ausência de associação espacial local. Assim, deve-se fazer uso de uma aleatorização condicional, que permitia determinar pseudoníveis de significância.

Para a obtenção de uma distribuição empírica das estatísticas de teste, deve-se observar se o valor da variável de interesse está dentro ou fora da região critica definida. Dessa maneira, se o valor calculado for superior em magnitude à esperança matemática do $I$ de Moran, seus resultados serão estatisticamente significativos.

\subsection{DESCRIÇÃO DOS DADOS}

As fontes de dados são do Instituto Brasileiro de Geografia e Estatística (IBGE), do Ministério do Desenvolvimento Social (MDS); Matriz de Desenvolvimento Social e do Instituto Paranaense de Desenvolvimento Social (IPARDES). Os dados utilizados são: benefício médio das famílias beneficiadas pelo PBF nos municípios do Estado do Paraná nos anos de 2004, 2008 e de 2012 e a taxa 
BRAMBILLA, M. A.;MARCONATO, M.;CÂRMARA, M. R. G.;CARVALHO S.C.;NASCIMENTO, S.P. Progama bolsa família: Uma análise espacial nos...

de pobreza dos municípios do Paraná.

O benefício médio das famílias do Programa Bolsa Família (BMPBF) refere-se ao valor anual total repassado em reais ( $\mathrm{R} \$$ ) aos beneficiários do Programa Bolsa Família dividido pela quantidade total de famílias beneficiadas em cada município.

Para estimar o número de famílias por município no Estado do Paraná nos anos de 2004, 2008 e 2012, foi utilizada a variação da população estimada pelo Instituto Brasileiro de Geografia e Estatística (IBGE, 2015).

Para identificar as famílias pobres nos municípios do Paraná dividiu-se o número de famílias beneficiárias pelo PBF pela taxa de cobertura do programa do MDS (2015).

Na Tabela 2 está apresentada à taxa de pobreza para cada ano analisado e os respectivos valores utilizados pelo Ministério do Desenvolvimento Socia (MDS) para conceder o beneficio do Programa Bolsa Família. Para encontrar a taxa de pobreza nos municípios paranaenses nos anos 2004, 2008 e 2012, dividiu-se a quantidade de famílias pobres pelo total de famílias estimadas para os municípios e multiplicou-se por 100.

TABELA 2: VARIÁVEL TAXA DE POBREZA E RESPECTIVOS VALORES DE REFERÊNCIAPARAOPROGRAMABOISAFAMIIANOSANOSDE 2004, 2008 EDE2012

\begin{tabular}{|c|l|}
\hline Variável & \multicolumn{1}{|c|}{$\begin{array}{c}\text { Linha de Pobreza - Valores de referência do } \\
\text { (PBF) }\end{array}$} \\
\hline $\begin{array}{c}\text { Taxa de } \\
\text { pobreza no ano } \\
\text { de } 2004\end{array}$ & $\begin{array}{l}\text { Refere-se às famílias com renda igual ou menor do } \\
\text { que R\$ 100,oo per capita }\end{array}$ \\
\hline $\begin{array}{c}\text { Taxa de } \\
\text { pobreza no ano } \\
\text { de 2008 }\end{array}$ & $\begin{array}{l}\text { Refere-se às famílias com renda igual ou menor do } \\
\text { que R } \$ 120,00 \text { per capita }\end{array}$ \\
\hline $\begin{array}{c}\text { Taxa de } \\
\text { pobreza no ano } \\
\text { de 2012 }\end{array}$ & $\begin{array}{l}\text { Refere-se às famílias com renda igual ou menor do } \\
\text { que R } \$ 140,00 \text { per capita }\end{array}$ \\
\hline
\end{tabular}

Fonte: Elaborado pelos autores com informações do MDS (2015)

\section{ANÁLISE DOS RESULTADOS}

Nesse item é realizada a análise exploratória de dados espaciais das variáveis taxa de pobreza e benefício médio (PBF) do Programa Bolsa Família para os municípios do Paraná.
BRAMBILLA, M. A.;MARCONATO, M.;CÂRMARA, M. R. G.;CARVALHO, S.C.;NASCIMENTO, S.P. Progama bolsa família: Uma análise espacial nos...

\subsection{AUTOCORRELAÇÃO ESPACIAL GLOBAL BIVARIADA}

Na Tabela 3 é possível observar o resultado para autocorrelação espacial entre a taxa de pobreza e o Programa Bolsa Família nos anos de 2004, 2008 2012. Foi utilizada uma matriz de pesos espaciais do tipo Rainha (Queen). Como o valor I de Moran ficou acima do valor esperado 2 E (I) $=[-1 /(399-$ 1) $=-0,0025]$ nos anos analisados pode-se inferir que existe autocorrelação espacial positiva entre taxa de pobreza e benefício médio (PBF) municipal a um nível de significância de 1\%TABELA

3: AUTOCORRELAÇÃO ESPACIAL BIVARIADA - TAXA DE POBREZA E BENEFICIO MÉDIO PBF - 2004, 2008 E 2012

\begin{tabular}{c|c|c|c}
\hline Variável & Convenção & I de Moran & Probabilidade (\%) \\
\hline & & & \\
TX_POB_04w/W_BEN_MED_04 & & 0,1416 & 0,001 \\
TX_POB_08w/W_BEN_MED_08 & Rainha & 0,1506 & 0,001 \\
TX_POB_12w/W_BEN_MED_12 & & 0,3101 & 0,001 \\
& & & \\
\hline
\end{tabular}

O sinal positivo da estatística I de Moran da Tabela 3 indica que os municípios com elevada taxa de pobreza são cercados por municípios com elevado valor do PBF e as localidades onde a taxa de pobreza está abaixo da média são circunvizinhados por unidades municipais com baixo valor do PBF. Também sinaliza que os dados concentram no primeiro quadrante (Alto-Alto) e no terceiro quadrante (Baixo-Baixo)

A autocorrelação positiva entre a pobreza e o PBF foi observada em todos os períodos, entretanto, houve um aumento entre os anos de 2008 e 2012. Esse resultado indica um aumento na similaridade entre as duas variáveis nos municípios paranaenses, ou seja, o valor do beneficio médio aumentou mais nas regiões pobres e pode ter crescido menos nas localidades com menos pobreza.

$\mathrm{O}$ aumento na autocorrelação pode ter sido influenciado pelo crescente número de famílias atendidas pelo PBF no estado do Paraná, que aumentou em $22 \%$ entre os anos de 2008 e 2012, passando de 367 mil para aproximadamente 449 mil famílias, no mesmo período os recursos financeiros aumentaram em 90\%, passando de R \$ 332 milhões para R \$ 634 milhões. Entre os anos de 2004 e 2008 à quantidade de famílias beneficiarias aumentou em 19\%, e o valor total repassado as famílias pobres cresceu $142 \%$.

O aumento dos recursos financeiros do PBF destinado às famílias mais pobres, a inclusão de novos benefícios a partir de 2008 (ver tabela 1) e o aumento da

2.A esperança do I de Moran é dada por: [-1/ (n-1)], onde n é o número de municípios.

Revista de Economia, v. 40, n. 3 (ano 38), p. 47-67, set/dez. 2014. Editora UFPR 
taxa de cobertura do $\mathrm{PBF}^{3}$ são fatores que também podem ter contribuído para aumentar o valor do beneficio médio recebido pelos beneficiários em municípios com taxa de pobreza acima da média e aumentar a autocorrelação entre as variáveis nos municípios.

Outro fator que pode ter contribuído para o aumento da relação espacial entre as variáveis pobreza e Bolsa Família no ano de 2012 pode ter sido a melhor identificação das pessoas em situação de miséria nos municípios. Sobre o tema Paiva, Falcão e Bartholo (2013) destacam que a reformulação do formulário de cadastramento no cadastro único no ano 2009 permitiu identificar melhor as famílias em situação de pobreza. A implantação do novo formulário demandou o desenvolvimento de material instrucional específico e um longo processo realizado em Brasília, de qualificação de instrutores indicados pelos Estados, que capacitaram, entre 2009 e 2013, quase 30 mil entrevistadores do Cadúnico de todos os municípios do país. Este foi um investimento fundamental que proporcionou qualidade no momento da coleta da informação das famílias.

Segundo Haddad e Pimentel (2004), a análise da estatística I de Moran permite conhecer a forma de agrupamento dos regimes espaciais, entretanto, não torna possível a visualização de onde estão esses regimes e a sua evolução ao longo do tempo. Para isso será utilizado o diagrama de dispersão de Moran bivariado.

\subsection{DIAGRAMA DE MORAN BIVARIADO}

Na Figura 3 é apresentado o diagrama de Moran que exibe no eixo horizontal taxa de pobreza e no eixo vertical o beneficio médio do Programa Bolsa Família nos anos de 2004, 2008 e de 2012. O valor positivo do coeficiente I de Moran em todos os anos indica que a curva de regressão é positivamente inclinada.

No primeiro quadrante do diagrama estão concentrados os municípios exibem elevada taxa de pobreza e os vizinhos apresentam valor do beneficio médio do PBF elevado. No segundo quadrante estão os municípios que apresentam taxa de pobreza abaixo da média e são circunvizinhados por municípios com elevado valor do beneficio médio do PBF.

O terceiro quadrante corresponde aos municípios com taxa de pobreza abaixo da média e os vizinhos apresentam baixo valor do beneficio médio do PBF. No quarto quadrante estão localizados os municípios pertencentes a agrupamentos espaciais com elevada taxa de pobreza e cercados por vizinhos com valor do PBF abaixo da média.

3 A taxa de cobertura média no total dos 399 municííos do estado do Paraná passou de $74 \%$ no ano de 2004 para próximo de 89\% nos anos de 2008 e 2012 (MDS, 20
FIGURA 3. DIAGRAMA DE MORAN BIVARIADO - TAXA DE POBREZA E BENEFICIO MÉDIO DO PBF NOS ANOS DE 2004, 2008 E DE 2012

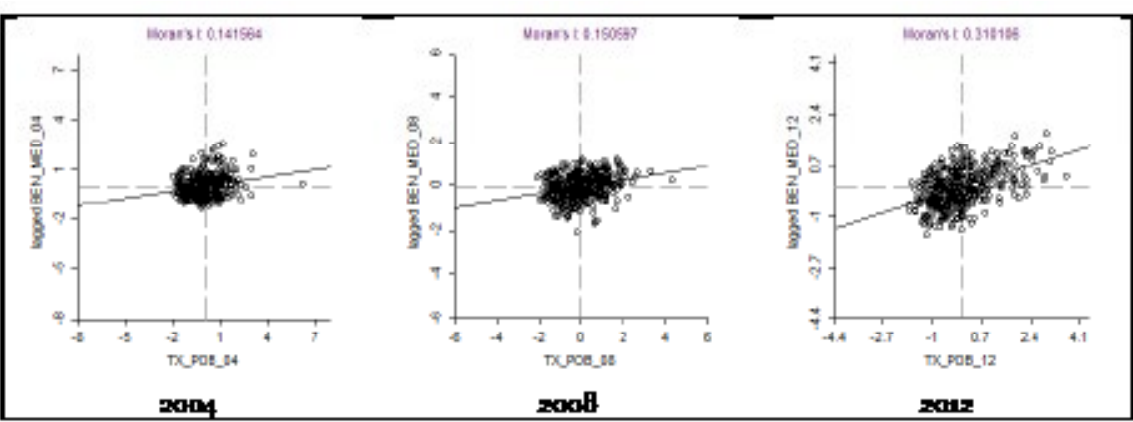

Fonte: Elaborado pelos autores, a partir de dados do IBGE e IPARDES

Conforme apresentado na figura 3, observou-se aumento da concentração espacial da taxa de pobreza e do benefício médio (PBF) dos municípios do Paraná ao longo do período 2004 a 2012. Assim, os municípios se encontram no primeiro e terceiro quadrante, evidenciando incremento da relação positiva entre as duas variáveis.

A disposição dos municípios no diagrama de Moran teve a seguinte distribuição para o ano de 2004, em torno de $17 \%$ das unidades municipais está concentrada no primeiro quadrante, configurando uma associação espacial alto-alto (AA), aproximadamente $36 \%$ estão situados no terceiro quadrante, denotando a presença de um regime espacial baixo-baixo (BB). No que se refere às regiões atípicas representadas pelo segundo e quarto quadrante, foi observado que $22 \%$ representam associação espacial baixo-alto (BA) e outros $25 \%$ são regime espacial alto-baixo (AB).

No ano de 2008, aproximadamente 29\% dos municípios analisados estão no primeiro quadrante, ou seja, alto-alto (AA) e $34 \%$ se concentram no terceiro quadrante, caracterizados como regime espacial baixo-baixo (BB). Nas regiões atípicas, $19 \%$ dos municípios se encontram no segundo quadrante, ou seja, aglomeração espacial baixo-alto (BA) e em torno de $18 \%$ das unidades municipais está situada no quarto quadrante, ou seja, alto-baixo (AB).

No que tange ao ano de 2012, foi observado que $28 \%$ dos municípios se encontram no primeiro quadrante, ou seja, alto-alto (AA) e em torno de $42 \%$ no terceiro quadrante, caracterizado como baixo-baixo (BB). Nas regiões atípicas, aproximadamente $18 \%$ estão no segundo quadrante, denotados como baixo-alto (BA) e em torno de $12 \%$ estão concentrados no quarto quadrante, sendo assim, regime espacial alto-baixo $(\mathrm{AB})$.

Os resultados observados nos diagramas de Moran (Figura 3) mostram que houve um incremento na quantidade de municípios localizados no terceiro quadrante, ou seja, caracterizados como baixo-baixo (BB) entre os anos de 
2012 e de 2008, e uma redução no número de unidades espaciais localizadas no quarto quadrante, denotadas como sendo regime alto-baixo (AB). Tal evidencia sugere que pode ter ocorrido uma redução da taxa de pobreza em diversas regiões entre o período 2012 e 2008 levando a um agrupamento dos municípios mais no terceiro quadrante, reforçando assim as informações disponíveis no IPEA (2015) onde é possível observar que houve redução de 35\% no número de domicílios pobres entre 2008 e 2012 no estado do Paraná. Paiva, Falcão e Bartholo (2013) reforçam que a expansão e focalização do Programa Bolsa Família foi um passo importante na consolidação de um sistema de proteção social que tem permitido ao Brasil reduzir a pobreza.

Os resultados encontrados corroboram os achados de Lima, Caldarelli e Camara (2014) que identificaram nos anos 2000, clusters de subdesenvolvimento, que concentravam a população mais pobre e as regiões menos desenvolvidas nas mesorregiões paranaenses no Centro Sul e Centro Oriental. Nesta direção, pode-se concluir que a focalização do programa e a melhoria dos instrumentos de coleta de dados ampliaram o alcance e os resultados do PBF no estado do Paraná, contribuindo para reduzir a pobreza e realizar a justiça social.

\subsection{AUTOCORRELAÇÃO ESPACIAL LOCAL BIVARIADA}

Mapeando os valores da probabilidade de medida, estatisticamente significativos, é possível gerar o mapa de significância de Moran local bivariado. A Figura 4 apresenta os mapas de significância das unidades espaciais através da estatística I de Moran bivariada local para as variáveis taxa de pobreza e benefício médio (PBF) nos anos de 2004, 2008 e 2012. No mapa, os municípios destacados em verde mais escuro representam os níveis de significância mais elevados (podendo variar entre 0,01\% a 5\%), as regiões destacadas em cor branca nãos são significativas.

FIGURA 4: MAPAS DE SIGNIFICÂNCIA BIVARIADA DA TAXA DE POBREZA E BENEFICIO MÉDIO DO PBF PARA OS ANOS DE 2004, 2008 E 2012

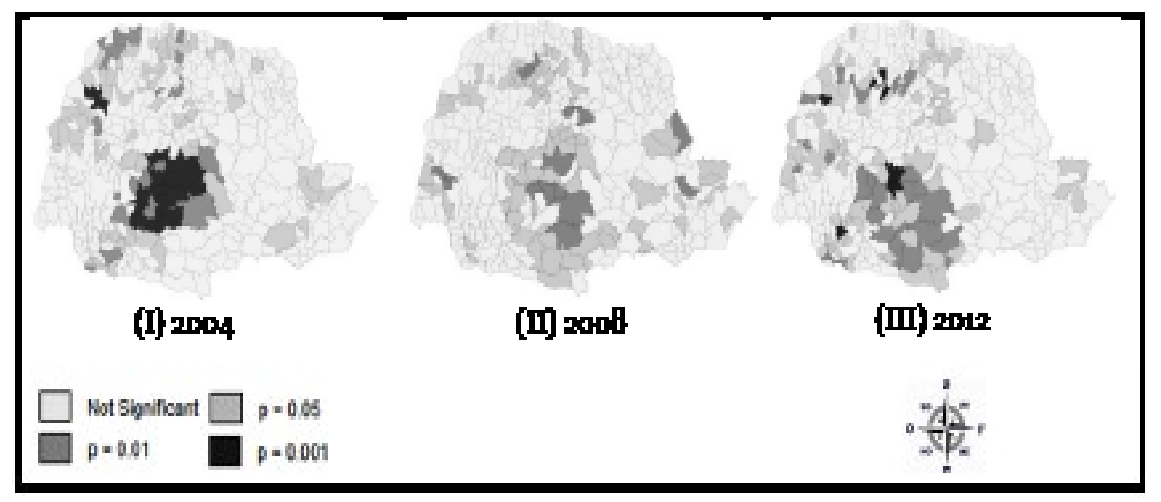

Fonte: Elaborado pelos autores, a partir de dados do IBGE e IPARDES
Na Figura 5 são apresentados os mapas de cluster bivariado que destacam onde foram formadas as aglomerações espaciais estatisticamente significativas divididas em quatro categorias de associação espacial. As localidades destacadas em preto representam os clusters espaciais alto-alto (AA), as unidades destacadas na cor cinza claro no mapa exibem os regimes espaciais baixo-baixo (BB), em cinza escuro está o cluster alto-baixo (AB) e em cinza estão em destaque às unidades municipais baixo-alto (BA).

No mapa pertinente ao ano de 2004 é possível observar a formação de cluster do tipo alto-alto (AA) predominantemente na região Centro-Sul, em torno de 32 municípios compõem o agrupamento com elevada taxa de pobreza e elevado Beneficio médio do PBF. Nas regiões Noroeste, Oeste e Norte Central se observou agrupamentos espaciais do tipo baixo-baixo (BB), nos quais aparecem 35 unidades espaciais que contemplam baixa taxa de pobreza e do valor do PBF.

No mapa referente ao ano de 2008, é possível observar à formação de cluster alto-alto (AA) formado por 27 municípios pertencentes à região Centro-Sul e Norte Central paranaense. Outro agrupamento composto por sete municípios com elevada pobreza e beneficio médio do PBF aparece destaque na mesorregião Sudeste. Os clusters do tipo baixo-baixo (BB) englobam 27 unidades espaciais localizadas principalmente nas regiões Norte Central e Noroeste, nesse ano também, aparecem três municípios denotados em cinza

claro na região Centro Oriental, outros sete municípios estão em destaque distribuídos pelo mapa.

Já, no mapa de 2012, é possível observar um cluster do tipo alto-alto (AA) formado por 28 municípios pertencentes às regiões Centro-Sul e Sudoeste, outro agrupamento composto por nove unidades municipais com elevada pobreza e beneficio médio do PBF aparece em destaque no Sudoeste paranaense. Três municípios caracterizados como alto-alto (AA) aparecem na região Metropolitana de Curitiba. No que tange as aglomeraç̃es espaciais do tipo baixo-baixo (BB), essas foram formadas por 51 municípios localizados principalmente nas regiões Norte Central, Noroeste e Oeste.

No ano de 2012 houve um aumento no número de municípios do tipo alto-alto (AA) e baixo-baixo (BB) em relação ao ano de 2004, a quantidade de município com elevada taxa de pobreza e beneficio médio do PBF passou de 32 no ano de 2004 para 40 no ano de 2012. Por outro lado, as unidades espaciais que contemplam baixa percentual de pobreza e do valor do PBF passaram de 35 para 51 no mesmo período.

Na região Sudeste é possível observar um acréscimo das unidades espaciais caracterizadas por taxa de pobreza acima da média e com elevado valor do beneficio médio do PBF, essas regiões denotadas em vermelho indicam que naquela região o programa assistencial Bolsa Família passou a atender mais 
BRAMBILLA, M. A.;MARCONATO, M.;CÂRMARA, M. R. G.;CARVALHO S.C.;NASCIMENTO, S.P. Progama bolsa família: Uma análise espacial nos...

famílias.

FIGURA 5: MAPAS DE CLUSTER BIVARIADO DA TAXA DE POBREZA E BENEFICIO MÉDIO DO PBF NOS ANO DE 2004, 2008 E 2012

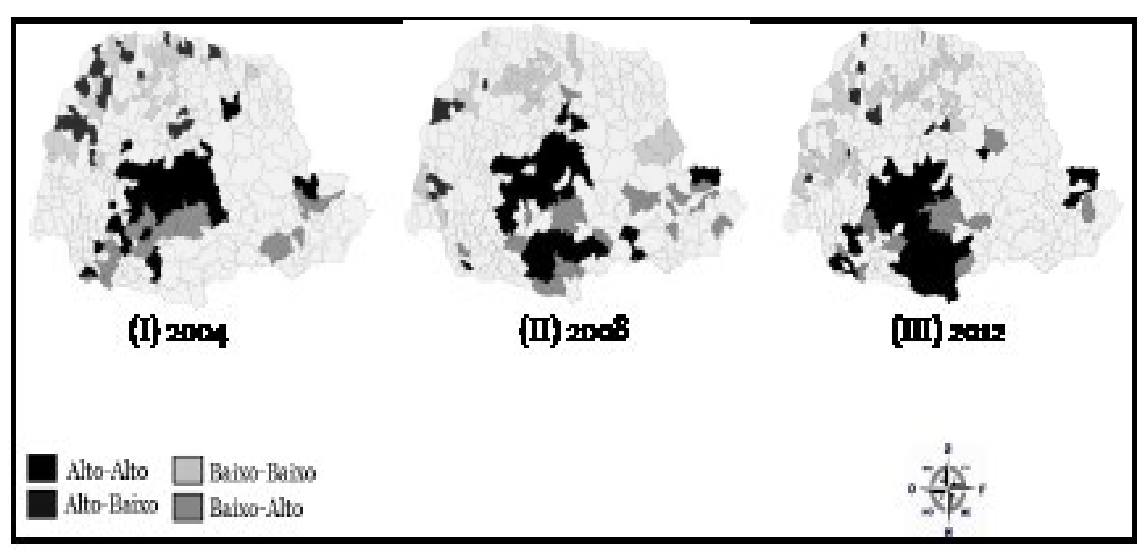

Fonte: Elaborado pelos autores, a partir de dados do IBGE e IPARDES

As informações obtidas a partir do I de Moran, do diagrama de dispersão e do mapa de cluster mostram que as regiões que exibem elevada taxa de pobreza são contempladas com maiores valores do benefício médio do Programa Bolsa Família. Assim há evidências de que o programa assistencial (PBF) está focalizado em regiões com maiores percentuais de pessoas vivendo em situação de pobreza. O resultado converge com as evidências encontradas em outros estudos que abordaram o tema como Jannuzzi, Bichir e Ritzi (2012) e Medeiros Britto e Soares (2007).

Os resultados dos diagramas (Figura 3) mostram que entre os anos de 2004 e 2008 houve um acréscimo de 48 municípios localizados no primeiro quadrante, ou seja, do tipo alto-alto (AA). Já, para o período entre 2012 e 2008 verificou-se o crescimento de 33 unidades municipais localizadas no terceiro quadrante, caracterizadas assim, como agrupamento baixo-baixo (BB), sinalizando a ampliação e a maior focalização do programa corroborando resultados de outros estudos anteriores sobre o tema, a exemplo de Lavinas (2006, 2007), Jannuzzi, Bichir e Ritzi (2012) e Medeiros, Britto e Soares (2007).

\section{CONSIDERAÇÕES FINAIS}

Esse estudo teve como objetivo verificar a relação entre taxa de pobreza e benefício médio (PBF) para os municípios do Estado do Paraná nos anos de 2004, 2008 e 2012, buscando identificar se os municípios com elevado percentual de pessoas vivendo na pobreza eram contemplados com maiores
BRAMBILlA, M. A.;MARCONATO, M.;CÂRMARA, M. R. G.;CARVALHO, S.C.;NASCIMENTO, S.P. Progama bolsa família: Uma análise espacial nos...

valores do beneficio médio (PBF).

Utilizou-se o coeficiente I de Moran e foi possível identificar autocorrelação positiva nos três anos analisados. Esse resultado indica que os municípios com elevada taxa de pobreza são vizinhos de municípios com alto benefício médio (PBF), por outro lado, os municípios com baixa taxa de pobreza são cercados por municípios com baixo benefício médio (PBF). No decorrer dos anos houve avanço da autocorrelação positiva entre a taxa de pobreza e o benefício médio (PBF) para os municípios no Estado do Paraná, principalmente entre os anos de 2004 e 2012, onde foi observado um aumento expressivo de 0,1506 para 0,3101. Houve um leve crescimento da concentração espacial no ano de 2008, quando comparado com o ano de 2004.

O resultado da análise de cluster espacial local revelou a existência de municípios com elevada taxa de pobreza e beneficio médio (PBF) na região Centro-Sul. As regiões Oeste, Noroeste e Norte Central, por sua vez, concentram aglomerações espaciais com baixa taxa de pobreza e beneficio médio do (PBF). Ao longo do período analisado foi possível verificar um aumento no decorrer dos anos no número de municípios do tipo alto-alto e baixo-baixo, e consequentemente uma redução das regiões atípicas (alto-baixo e baixo-alto).

$\mathrm{O}$ resultado do estudo reforça as constatações encontradas nos estudos de Lavinas (2005, 2006, 2007) e Jannuzzi, Bichir e Ritzi (2012) que mostram que os programas de transferência de renda, trazem grandes benefícios para a população mais vulnerável. A ampliação e boa focalização dos programas tende a contribuir para a redução da pobreza. A ampliação de benefícios do Programa Bolsa Família, principalmente com benefício de superacão da extrema pobreza (BSP) com início em 2012, garante a todas as famílias atendidas pelo programa uma renda para a superação da extrema pobreza, elevando o valor do benefício médio em cidades com altos índices de pobreza. O estudo permite concluir que houve expansão do programa no Paraná e que ele alcançou as regiões mais pobres do estado.

\section{REFERÊNCIAS}

ALMEIDA, E. Econometria Espacial Aplicada. Alínea: Campinas: SP, 2012.

ANSELIN, L. Spatial Econometrics: methods and models. Boston: Klumer academic, 1988.

Local indicators of spatial association - LISA. Geographical Analysis, v. 27, n. 2. p. 93-115, 1995 .

Interactive techniques and exploratory spatial data analysis. In: LONGLEY, M. F.; GOODCHILD, D. J.; MAGUIRE, RHIND, D. W. Geographical information systems: principles, techniques, management and Applications. New York: Jonh Wiley. p. 251-264, 1999.

SYABRI, I.; SMINORV, O. Visualizing multivariate spatial correlation

Revista de Economia, v. 40, n. 3 (ano 38), p. 47-67, set/dez. 2014. Editora UFPR 

BRAMBILLA, M. A.;MARCONATO, M.;CÂRMARA, M. R. G.;CARVALHO,
S.C.;NASCIMENTO, S.P.Progama bolsa família: Uma análise espacial nos...

with dynamically linked windows. University of Illinois, 2003.

BARROS, R. P.; CARVALHO, M. Desafios para a Política Social Brasileira Rio de Janeiro: IPEA, texto para discussão, n. 985, out. de 2003.

HENRIQUES, R.; MENDONÇA, R. A Estabilidade Inaceitável: Desigualdade e Pobreza no Brasil. Rio de Janeiro, IPEA, texto para discussão, n. 800, junho de 2001.

CARVALHO, M.; FRANCO, S.; MENDONÇA, R. A importância das cotas para a focalização do Programa Bolsa Família. Niterói, UFF texto para discussão, n. 238, agosto de 2008 .

CAMPELLO, T. Uma década derrubando mitos e superando expectativas. In. CAMPELLO, T.; NERI, M. C. Programa Bolsa Família: uma década de inclusão e cidadania, Brasília: Ipea, 2013.

COSTA, D. J.; CUNHA, M. S. O Programa Bolsa Família e a Oferta de Trabalho Evidências para o Brasil e Paraná. Revista de Economia, v. 40, n. 2 (ano 38) p. 105-128, mai./ago. 2014.

COTTA, T. C.; PAIVA, L. H. O Programa Bolsa Família e a proteção social no Brasil. In: CASTRO, J. A.; MODESTO, L. Bolsa Família 2003-2010: Avanços desafios. Brasília: IPEA, 2010. Cap. 2. p. 57-100.

DINIZ, S. S. Análise espacial da produtividade de laranja dos municípios do estado de São Paulo: 2002, 2010 e 2012. 118 f. Dissertacão (Mestrado Economia Regional) - Universidade Estadual de Londrina. Londrina, 2012.

FERNANDES, R. G. Programa Bolsa Família: Promoção de Cidadania? 2006. Web.intranet.ess.ufrj.br. Disponível em: <http://web.intranet.ess.ufrj.br/monografias/102053814.pdf>. Acesso em: 25 mar. 2015.

HADDAD, E. A.; PIMENTEL, E. A. Análise da distribuição espacial da renda no estado de Minas Gerais: uma abordagem setorial. Usp.br São Paulo 2004. Disponível em: <http://www.usp.br/nereus/wp-content/uploads/ TDNereus 02 04.pdf $>$. Acesso em: 04 out. 2013

IBGE - INSTITUTO BRASILEIRO DE GEOGRAFIA E ESTATÍSTICA. Disponível em: <http://www.ibge.gov.br >. Acesso em: 18 set. 2015

IPEA - INSTITUTO DE PESQUISA ECONÔMICA APLICADA. Disponível em $<$ http://www.ibge.gov.br>. Acesso em: 18 set. 2015.

JANNUZZI, P.; BICHIR, R.; RITZI, R. Bolsa Família e seus impactos nas condições de vida da população brasileira: principais resultados da pesquisa Avaliação do Impacto do Bolsa Família II. Brasília, 2012. Estudo técnico, n. 03/2012.

LAVINAS, L Pobreza no Brasil: números, miradas e miragens. IE. UFRJ. BR, 2005. Disponível em: < http://www.ie.ufrj.br/aparte/pdfs/lavinas_deficit_e redistribuicao.pdf $>$. Acesso em 04 out. 2013

Transferências de renda: o "quase tudo" do sistema de proteção social brasileiro. Anais do Encontro da ANPEC. 2006. Acesso em 4 de setembro de 2015 .

. Gasto social no Brasil: programas de transferência de renda versus investimento social. Ciência $e$ saúde coletiva. vol. 12, n.6, pp. 1463-1476. 2007.
BRAMBILLA, M. A.;MARCONATO, M.;CÂRMARA, M. R. G.;CARVALHO, S.C.;NASCIMÉNTO, S.P. Progama bolsa família: Uma análise espacial nos...

LIMA, V. M. A.; CALDARELLI, C. E.; CAMARA, M. R. G. Análise de desenvolvimento municipal paranaense: Uma abordagem espacial para a década de 2000. Revista Economia e Desenvolvimento. v. 26, n. 1, p. 1-19, 2014.

MARINHO, E.; LINHARES, E.; CAMPELO, G. Os programas de transferência de renda do governo impactam a pobreza no Brasil? Scielo.br. 2011. Disponível em: < http://www.scielo.br/scielo.php?script=sci_arttext\&pid =So034-71402011000300003>. Acesso em 04 out. 2013.

MEDEIROS, M ; BRITTO, T ; SOARES, $\mathrm{F}$ Transferência de renda no Brasil. Scielo.br. 2007. Disponível em: http://www.scielo.br. Acesso em 04 out. 2013

MDS - MINISTÉRIO DO DESENVOLVIMENTO SOCIAL E COMBATE À FOME. Disponível em: < www.mds.gov.br>. Acesso em 16 set. 2015.

MONTEIRO, A. A. D. Alocação de recursos e eficiência na gestão do Programa Bolsa Família. Dissertação (mestrado em administração) - 2008. 10 f. Universidade Federal de Viçosa. Viçosa, 2008.

NETO, V. R. P; MEDEIROS, C. N. Análise espacial da extrema pobreza no estado do Ceará. Texto para discussão, Instituto de Pesquisa Estratégica Econômica do Ceará (IPECE), Fortaleza, 2011.

OLIVEIRA, L. F. B., SOARES, S. S. D. O que se sabe sobre os efeitos das transferências de renda sobre a oferta de trabalho. Rio de Janeiro, IPEA, texto para discussão, n. 1738, maio de 2012.

PAIVA, L. H.; FALCÃO, T.; BARTHOLO, L. Do Bolsa Família ao Brasil sem miséria: um resumo do percurso brasileiro recente na busca da superação da pobreza extrema. Programa Bolsa Família: uma década de inclusão e cidadania, Brasília: IPEA, 2013.

PIRES, D. C. A., LONGO, L. A. F. B. A implantacão do Bolsa-Família e sua relação com a pobreza nas Regiões Brasileiras no período de 2004 e 2006. Abep.org.br, 2008. Disponível em: <http://www.abep.nepo.unicamp. $\mathrm{br} /$ encontro2008/docsPDF/ABEP2008 1145.pdf $>$. Acesso em: 04 de outubro de 2013

SABATER, L A.; TUR, A. A · AZORÍN, J. M. N. Análise Exploratória dos Dados Espaciais. In: COSTA, J.S.; DENTINHO, T.P.; NIJKAMP, P. Compêndio de Economia Regional: métodos e técnicas de análise regional. Vol. 2. Principia, p. 237-298, 2011.

SILVA, J. G.; TAVARES, L Sobre o futuro do bolsa família. In CASTRO, J. A. MODESTO, L. Bolsa Família 2003-2010: Avanços e desafios. v.2, Brasília: IPEA, 2010. Cap. 1. p. 235-265.

SILVA, M. O. S.; YAZBEK, M. C.; GIOVANNI, G. A Política Social Brasileira no Século XXI: a prevalência dos programas de transferência de renda. São Paulo: Cortez, 2004.

SOARES, S.; SÁTIRO, N. O Programa Bolsa Família: Desenho institucional e possibilidades futuras. In: CASTRO, J. A.; MODESTO, L. Bolsa Família 20032010: Avanços e desafios. Brasília: IPEA, 2010. Cap. 1. p. 25-56 